\title{
Healthy promotion: changing behaviour towards evidence-based health care
}

Department of Psychology, University of Leeds, Leeds LS2 9JT, UK

Jenny Firth-Cozens, principal research fellow in clinical audit

Accepted for publication 18 August 1997

Jenny Firth-Cozens

All organisations have to change to meet challenges or take forward new opportunities. Health organisations are no different; all over the world they face pressures to buy and to provide care that is based on evidence of its effectiveness. This is not a new concept: it is what staff within those organisations have always considered themselves to be doing. What is new is the emphasis that is put upon the move towards a truly evaluative culture, one which asks systematically and regularly:

- Is there evidence to back up the effectiveness of this procedure or show another to be superior?

- Where can I find out about it?

- How can I let my team know about it?

- Are we applying it?

- Are our outcomes as good as can be expected?

All this necessitates an increase in certain behaviours involving the seeking for information about good evidence; communicating it within the organisations; applying it wherever appropriate; and monitoring its applications and outcomes. This paper explores the issues to be considered by those encouraging the development of this evaluative culture, looking for leads from research in other areas of work involving behaviour change: organisational and health psychology, health promotion, and marketing.

\section{Context}

To bring about major changes within organisations, it is also essential to appreciate the importance of the wider culture and context to understand some of the forces that may hinder or hasten the change. The move towards clinical and cost effectiveness is taking place around the world against a background of major restructuring of health services, a move towards explicit rationing, the introduction of increasingly specialised new technology, a fall in the status of the professional, and the presence of high unemployment. This backdrop of current economic and social pressures adds to historical ones concerning old tensions between groups and the special difficulties and demands of the job of caring for patients. ${ }^{1}$

Those responsible for change in such complex organisations are often urged to concentrate on the natural innovators and enthusiasts rather than directing their energies towards "laggards". ${ }^{2}$ It may well make sense to have opinion leaders on board ${ }^{3}$ where possible; however, ignoring those who seem hard to shift inevitably creates an increasing gap in practice between those who are favoured both with an innovative personality (and quite often with extra resources and education to extend their work towards the ideal) and the rest. This will lead in turn to an inevitable increase in the variation of healthcare behaviour and of the outcomes that result, something which is completely contrary to the ideas of clinical effectiveness. I would argue therefore that it is equally, if not more, important to try to understand and change those who find it difficult to shift rather than continuing to target those who change with ease.

The second reason for this broader focus is that in an arena of constant organisational change we are likely to see those who take up new things with alacrity as somehow superior to those who are less enthusiastic about change. However, most organisational experience informs us that diversity in these aspects of personality is often a benefit, with those who hold back acting as a safety anchor against decisions which are too fast or not well enough thought through. ${ }^{45}$ For these reasons this paper is directed as much towards affecting those slow to change as the rest. Its purpose is to break down the complexity of change into manageable chunks and so to provide a framework for the culture of dissemination and the use of good evidence. The table shows the questions for the dissemination framework.

\section{Knowing about it}

Dissemination of evidence-based health care involves getting information about what works and what does not to those concerned at all levels of the organisation. Although this knowledge is necessary, it is not sufficient to result consistently in a change in practice. ${ }^{6} 7$ Nevertheless it is an essential first step to make the product itself - the information that needs to be communicated-one which will attract rather than be ignored or even repel. To do this you need to be aware of whom you want to change, what their cultures, experiences, and motivations are, and what the characteristics of the product should be to make it most attractive.

\section{Who should change?}

If we use marketing language ${ }^{89}$ those whose behaviour we want to influence are the customers for our products, and the customers in an evidence-based approach are going to be primarily clinicians and patients. However, we need to be much more precise with our focus: despite health professionals sharing the common aim of providing good patient care, beyond this they may differ considerably, with various needs and motivations at different times in their careers and different levels and 
Table 1 Dissemination: the product and its promotion

Whom are you trying to change?
General practitioners
Consultants
Junior doctors
Non-medical professionals
Patients
Managers
Educationalists
Commissioners
Who else should be involved?
At which stage of change is the target group (and any others
who are involved)?
Precontemplation
Contemplation
Preparation
Change
Maintenance
So what should the characteristics of the products be to
encourage change?
Easy to try out
Delivering an obvious advantage
In line with prevailing norms and values
Able to be changed by staff involved
How and where will this be promoted?
By identifying gatekeepers of information
Through education
Backed by someone respected
Available where it will be attended to best
Be clearly in line with target group's motivations

types of knowledge due to a variety of educational paths. Social influence theory informs us that recognising and working with cultural differences-customs, beliefs, values, etc-is essential if we are to affect the ways that information is interpreted and acted on. ${ }^{10}$

For example, junior doctors may see change as an important aspect of their training or of their promotability; nurses might view it as an opportunity to learn how to search the evidence; consultants may regard it as a means to acquire new technology; commissioners may require it as a way to reduce expenditure; patient groups will view it as a means to empower patients; chief executives might use it to make their services offered more attractiveand so on. These motivational differences were demonstrated in a recent survey of members of the British Association of Medical Managers (BAMM) and general practitioners ${ }^{11}$ which asked, among other things, about aspects which may make an evidence based approach more likely for them to follow. Although both groups saw improvement in patient outcomes as the most important reason for change, half of the general practitioners and none of the BAMM members thought that financial incentives would help persuade them. By focusing on the motivations, values, costs, and rewards of a particular group we are much more likely to make change successful.

An important issue for dissemination of information about best practice will be the information needs of each particular group, and this will depend on their stage of change.

\section{Stages of change}

People are going to need different types of information at different stages of the dissemination and implementation process. Prochaska et $a l^{12}$ have described the importance in health promotion of targeting people according to their level of preparedness to change.

For example, there is still a sizable proportion of health professionals in the
United Kingdom who have not yet heard of the clinical effectiveness agenda nor of any of its helpful publications. ${ }^{13}$ In health promotion terms, these people are said to be precontemplation stage: they need to be told basic information about what the approach involves and why it matters so that they can start to think about what it might mean to their practice and what they need to do next. When they do this they are said to be in the contemplation stage, followed by the preparation stage, perhaps when they are learning to use their first CD-ROM, writing off for professional or patient association guidelines, or attending a critical appraisal skills workshop. If these preparations prove attractive enough, the next stage should be action, when they begin to change their practice as a result of the information; followed by maintenance, the stage where they need the occasional reminder or reinforcer to keep up the momentum. ${ }^{14}$ Without maintenance, any initial impact of even prominent national guidelines can decline. ${ }^{15}$ Different means of change, including different types of information, will be appropriate at each stage. ${ }^{16}$

\section{Marketing strategies}

In marketing terms, the means of bringing about a change in behaviour involves enhancing the design of the product, its promotion to the right people, and making its price as attractive as possible. ${ }^{17}$ In bringing about an evidence-based approach to health care, the products (and to some extent their promotion) include such initiatives as a patient information leaflet, a page on the Internet, a CD-ROM from the Cochrane Library, an audit report, national guidelines, a workshop to learn critical appraisal skills, a "quick sheet" accident and emergency guide, ${ }^{18}$ etc. Winett ${ }^{19}$ points out that research has demonstrated that, to bring about adoption, products need to:

- Be able to be tried without great cost or effort. It is important therefore to have them available in easily accessible places, within working hours, at times when they may be most salient, etc.

- Deliver a relative advantage. An advantage in improved patient care is something which is of the essence in both clinical audit and the clinical effectiveness programme, but needs to be shown clearly.

- Be able to be reinvented. Although this will not be a feature of all products, it is in line with the additional success that is sometimes achieved by the local adaptation of guidelines. $^{19}$

- Fit well with prevailing norms. This will involve working with the culture of the target group-what they care about, what their values involve. For example, as nursing begins to put its emphasis more on academic criteria rather than caring ones, the direction of emphasis of a product for them may need to change. For health professionals as a whole, it may be more acceptable to argue only for clinical effectiveness rather than cost effectiveness, as clinical effectiveness fits the clinical culture more comfortably. 
Promoting the evidence

Promotion of dissemination in all the appropriate ways and places is an essential step without which even prominent national guidelines will be unnoticed. ${ }^{20}$ For example, a successful change in practice to reduce $x$ ray films for ankle traumas involved various levels of instruction including a brief lecture, a handout, a pocket card, and two posters in the accident and emergency departments. ${ }^{21}$ There is a continuing task for educational initiatives at both undergraduate and postgraduate levels to provide information about the approach, and training in the core skills involved. In fact, this is transforming medical education in some places. $^{22}$ The effects of continuing medical education in changing actual practice seem less promising: a 1992 review of randomised controlled trials found no effects on healthcare outcomes of simply disseminating the evidence by this means. ${ }^{23}$ Nevertheless, more recent reviews and studies have shown varying benefits for continuing medical education-for example, a study to increase the use of prophylaxis for venous thromboembolism found a significantly greater shift in hospitals where physicians took part in a formal continuing medical education programme than in those who did not, although both groups improved, ${ }^{24}$ and adding a quality assurance element made no extra difference. A more recent review ${ }^{25}$ found that methods such as conferences had little impact, but $79 \%$ of those which used three or more educational strategies were effective in bringing about change, and in two studies this was followed by an improvement in patient outcomes.

Lessons from health promotion suggest that it is important who backs the products and the initiative as a whole, so the support of professional associations and the promotion by a local opinion leader is likely to be beneficial ${ }^{3}$ although this is not always the case. ${ }^{7} \mathrm{~A}$ recent survey showed that we are a long way from finding the right routes for information to reach the appropriate parts of the organisation. ${ }^{26}$ Dawson points out that, in the literature on technology transfer, there are always gatekeepers to information, ${ }^{8}$ and it will be equally important to identify who these are and to work with them on the most useful ways that they can help in the dissemination process.

In promoting any aspect of the approach, it is necessary to consider the particular motivations and stages of change of the group you are targeting within any promotional literature, and to emphasise points that will be salient to them. If these are not apparent, then the use of focus groups to discover particular concerns and needs should make the product and its promotion more successful. ${ }^{27}$ It is noticeable, for example, that new treatments (such as counselling in primary care ${ }^{28}$ or various costly medications) seem to be taken up swiftly often without adequate evidence of effectiveness, whereas others which involve stopping treatments or using simpler ones like aspirin seem more difficult to change. ${ }^{29}$ The psychology and values which underlie a need to do, rather than not to do, need to be considered during under- graduate training as well as in the promotion of particular products.

\section{Forces that help and hinder}

All change involves moving away from a state of equilibrium which is maintained by forces which drive forward and counter forces which restrain. ${ }^{30}$ Both directly and indirectly, these forces contribute towards the price of change: the costs to the individual person or the group, less the benefits that result. We change by altering the balance between the barriers and the drivers, either by increasing the drivers or reducing the forces which hold us back. Therefore, recognising and dealing with the organisational and individual barriers to change are essential. The figure sets out some of those forces which are likely to operate in introducing an evaluative culture. There will inevitably be others apparent within individual organisations, and it needs local work to discover their importance.

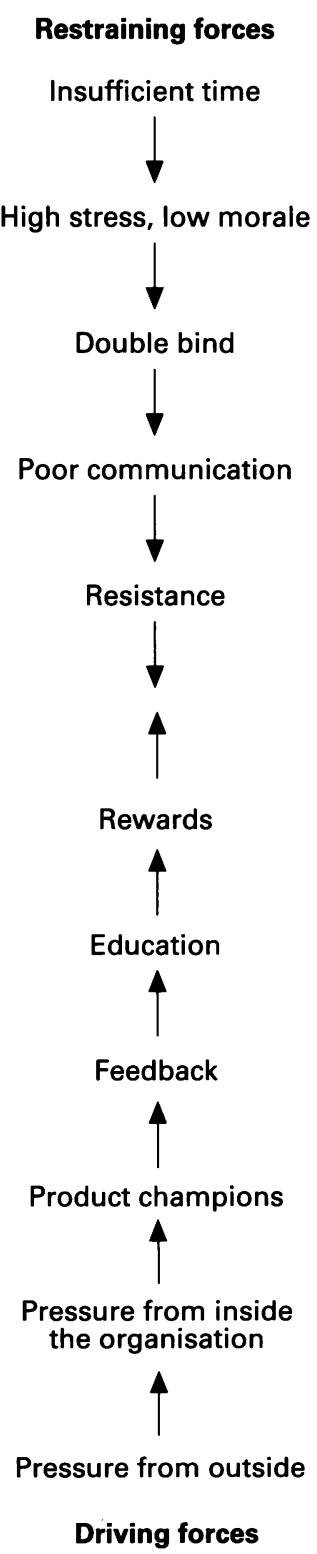

Forces that help and hinder an evidence-based approach. 
RESTRAINING FORCES

Insufficient time

The overriding cost to individual people of introducing another step into their work is the increased pressure on their time. With clinical staff already overburdened, the addition of lunchtime meetings, courses, library work, etc, is the primary concern of many staff in doing audit, and this will be no different in the development of an evidence-based approach. ${ }^{31}$ In fact, one of the greatest problems in bringing about an evaluative culture is the inevitable time it takes to bring different groups together: managers and clinicians, different specialties, primary and secondary care, purchasers and providers, and so on. ${ }^{32}$ Nevertheless, this is an essential part of the process, ${ }^{18}$ and unless time to consider these issues is carved out for staff, they will ignore it or, at best, pick it up and consider it in a piecemeal fashion, ready to drop as soon as the emphasis seems to shift away. Taking the time shortage seriously might involve general management working with clinicians on what it takes to create an organisation that is based on clinical and organisational effectiveness and how this can be achieved.

\section{High stress, low morale}

Stress is considerably higher in health workers than in other employees ${ }^{1}$ and high stress is linked to low morale and low job satisfaction as well as to deficits in performance, ${ }^{33}$ which often includes a reluctance to take on new things. Initiatives designed to reduce occupational stress are likely to increase participation and self efficacy, ${ }^{34}$ which in turn could influence a greater commitment towards the desired changes. Although it has been suggested that an evidence-based approach may lead to lower job satisfaction, ${ }^{35}$ it may equally be the case that being fully involved in and confident that you are giving the best health care will actually increase job satisfaction and reduce stress in itself.

\section{Territorialism}

Professionalism has many positive attributes, but it also necessarily involves the construction of boundaries within which special knowledge is thought to reside. The move towards evidence-based health care is threatening to any extremes of professional territorialism as it involves the open access to evidence which is equally available to other professional groups and to patients. The evaluative culture that is at its root will allow not only questioning by those involved in the profession but by commissioners, patients, and others. Involving professional bodies throughout the process of change is essential, but also helping clinicians to influence their professional associations in the other direction may be important too, as these bodies rightly exist to promote the interests of their members and some may need help to do this in new ways. The American Psychological Association, for example, has used an evidence-based approach to demonstrate to insurance companies and others the certain value of using clinical psychologists. ${ }^{36}$
Double bind

Trust is a crucial part of any change initiative. Policy and management need to be seen to be firmly committed and obviously in line with the clinical effectiveness agenda rather than ambivalent about the relative merits of activity and effectiveness so that each are put forward as essential in a double bind message. This can create confusion or cynicism in staff so that neither route may actually be followed. Using restraints and incentives to support change towards increasingly effective care rather than in ways which maintain the status quo needs to be done transparently. ${ }^{8}$

\section{Poor communication}

Communication, tailored to the needs of target groups, is at the heart of an evidence-based approach, and change will not occur if there is inadequate or inaccessible sources of information about what is clinically effective or ineffective. Good communication will also involve being clear about what is wanted, what will be rewarded, and how and why it matters, and so on. The more involvement there is in the process, the less chance there will be of clinicians and patients thinking that actions are imposed on them rather than arising through any genuine two way dialogue. ${ }^{837}$

\section{Resistance}

Resistance to change is almost always seen as a bad thing, irrational, and something to be overcome. But this presupposes that all change is good and should be accomplished by the fastest, least challenged means possible. Resistance is actually a necessary process and likely to be beneficial in the early days, a counterforce to unnecessary actions or to changes happening too fast or with insufficient thought. As I described earlier, there are some of us who enjoy change for change's sake, and some who dislike change and value the here-and-now. Because of our present culture, it is those who enjoy change who are likely to rise to the top of organisational structures, and this can make it hard for them to understand the apparent reluctance of others to take on board their enthusiasms. An appreciation of differences will help to create change that is better for its pace being slowed and its consequences better anticipated. ${ }^{4}$

There are, however, other aspects to resistance which are likely to be barriers to the emergence of an evidence-based culture. For example, anger at the imposition of what may be seen as still more demands may make even the most enthusiastic clinicians dig in their heels. Also, there is a real but unacknowledged implication that the introduction of clinically effective care means that clinicians may, up until then, have being delivering less than effective care. Linked to this is the fear of evaluation and criticism which many of us experience and which is a particular feature of those in the medical profession ${ }^{38}$ : no matter how much the approach is emphasised as nonjudgmental, the necessity of monitoring process and outcomes will arouse any underlying dislike of putting one's work up for scrutiny. 
The desire to provide rather than withhold treatments, discussed earlier, may also create resistance to particular changes.

These emotional responses and others, both conscious and unconscious, which make up resistance to change, cannot always be overcome. However, as every psychotherapist knows, they need to be acknowledged and this in itself will often take away some or all of their force.

\section{FORCES FOR CHANGE}

Rewards

The price of a product or an action is inevitably going to be an extremely important influence on its uptake. The price is the cost payments out or effort involved, less the rewards and benefits received. We know that evidencebased care will always initially require extra time; anything that can be done to reduce this will decrease the cost-for example, having information in appropriate and accessible places in user friendly designs, providing dedicated time for audit for all professional groups, making the whole experience unthreatening, and so on.

The other way to reduce the price of change is to increase the rewards received by change. Rewards, long recognised as powerful forces for encouraging new behaviours, can be:

- Financial-for example, through extra staff or, in the United States, because certain risk management strategies are rewarded by decreased insurance costs ${ }^{39}$

- Psychological-in increased job satisfaction, self esteem, appreciation, or a reduction of uncertainty ${ }^{40}$

- Educational-to do with learning new skills or having increased career prospects

- Social-for example, by being part of a team that is successful in providing the best care; increasing the sense of empowerment as a result of relevant information.

Exploring the precise rewards appropriate for particular groups might be the subject of surveys or focus groups. Although recent arguments have pointed out that reward systems may block innovative practice ${ }^{41}$ there is also compelling evidence that this is not inevitable. ${ }^{42}$ Moreover, an evidence-based approach is not an area of organisational behaviour where innovation is particularly appropriate. ${ }^{43}$ Nevertheless, the anti-reward arguments will warrant further consideration as they provide evidence of the tailing off of behaviour once reinforcement endssomething which may stop the hoped for embeddedness of an evidence-based or evaluative culture unless the rewards gained become internalised, psychological ones, rather than externally provided.

Learning also takes place through the withdrawal of rewards or even through punishments-such as the increase of insurance costs, the threat of litigation, or the loss of a service. However, an increase in court actions is not necessarily going to lead to more evidence-based health care and may even result in defensive practices. ${ }^{44}$
Finally, we need to ensure that we are not rewarding clinicians more in any way for not following a clinically effective guideline than we are for following it - for example, by rewarding increased quality less than we do greater activity in an intervention the benefits of which are doubtful.

\section{Education}

Education in traditional ways may not always show substantial benefits ${ }^{23}$ but several dissemination and education strategies linked to other forces for change are more likely to be successful. ${ }^{25}$ It may be that a piecemeal approach to education is not so useful in this regard, and we are better to change our undergraduate and postgraduate education more radically towards a problem solving approach that systematically incorporates the use of evidence. $^{22}$

\section{Feedback}

Feedback of monitoring data-for example, audit data-has had mixed results in bringing about change. Whereas Lomas et a ${ }^{\beta}$ found it less useful than the use of an opinion leader, others have reported large changes after the feedback of the audit cycle. ${ }^{45}$ It may be, however, that change through feedback occurs in the longer term only when the change is rewarding in some way to the individual. We know from psychological research that feedback is an important part of behavioural change, acquisition of new skill, and initiating and continuing desired behaviours, ${ }^{46}$ but it is likely that no one is going to maintain that new behaviour if it does not also have a reward intrinsic to it-for example, interest, career enhancement, belonging to a group, etc.

Opinion leaders, product champions, etc

Getting the right person to promote the product is an essential part of marketing and of health promotion and this will usually be someone who is respected or in authority. ${ }^{47}$ Equally important is to link them into the management system to ensure that their influence is in line with strategy and to make sure they are replaced should they leave. ${ }^{5}$ Perhaps because of the strength of the forces of resistance, there is always a tendency to revert to the original position even after good initial change ${ }^{43}$ and maintenance strategies will be necessary to ensure that this does not happen.

\section{Pressure from inside the organisation}

The idea that clinically effective care is the responsibility of only clinicians is unlikely to be acceptable or useful. Having in place a total quality system which uses evidence-both clinical and occupational-wherever possible in its decision making will be an important force for change. Within that organisation, teams can themselves be highly influential in encouraging clinically effective care because of the social rewards they provide to those who are committed members. ${ }^{5}$ This presupposes, however, that the leadership is respected and committed to an evidence-based approach as there is strong evidence that the group is not 
always as rational as the individual or as well behaved. ${ }^{43}$ Similarly, teams may not always listen well once they have embarked on a project. ${ }^{8}$ For this reason it is important that they should be linked into the management structure and should be clear about their role in producing a clinically effective organisation.

\section{Pressures from outside the organisation}

Nowadays forces for quality may come from numerous agencies outside the organisation: from commissioners, insurers, from professional associations, policy makers, and patients. There is as yet little evidence in the United Kingdom that policy is consistently evidence-based, nor that contracting is being used successfully as a tool for quality, ${ }^{48}$ although there are purchasers who are using other influences to bring about change through working together with local clinicians. ${ }^{49}$ Also, the slowly increasing voice of the patient, including the use of complaints and legal actions, may well become an important force for change in the future, although its effects may not be as obviously beneficial as is hoped: patients may make demands on the clinicians which actually curtail them from taking an evidence-based decision. ${ }^{50}$

\section{Conclusions}

There are several areas of research which focus on the factors that influence changes in behaviour and these should prove important in encouraging the development of an evaluative culture. The task is as complex as any other that involves human behaviour, and so the use of relevant strategies with targeted groups is likely to have the best chance of success. Whichever group is the focus for change, whether clinicians, or patients, or managers, the process will best be achieved through a genuine two way flow of information. This will be a very long term endeavour, constantly in need of further consultation and further adaptation to enable it to become a demand from the customers rather than simply a supply of information from above. Finally, the more the change strategies themselves are subjected to rigorous evaluation, the more the task of using good evidence can be spread across an organisation and across policy making itself.

1 Borrill CS, Wall TD, West MA, et al. Mental Health of the workforce in NHS trusts. Interim report to the National Health and Safety Executive, Northern and Yorkshire Region Research and Development Division. Durham: NHS Executive, 1996.

2 Stocking B. Promoting change in clinical care. Quality in Health Care 1992;1:56-60.

3 Lomas J, Enkin M, Anderson GM, et al. Opinion leaders vs audit and feedback to implement practice guidelines: delivery after previous caesarian section. $\mathscr{f} A M A$ 1991;265 2202-7.

4 Bridges W. The character of organisations: using fungian type in organisational development. Palo Alto: Consulting Psychologists Press, 1992

5 Firth-Cozens J. Building teams for effective audit. Quality in Health Care 1992;1:252-5.

6 Col NF, McLaughlin TJ, Soumerai SB, et al. The impact of clinical trials on the use of medications for acute myocardial infection: results of a community-based study. Archives of Internal Medicine 1996;156:54-7.

7 Oxman A, Thomson MA, Davis DA, et al. No magic bullets: an intervention to improve professional practice. Can Med an intervention to impri 8 Dawson S. Never mind solutions: what are the issues? Les-
sons of industrial technology transfer for quality in health sons of industrial technology transfer for qualit
care. Quality in Health Care 1995;4:197-203.

9 Dickinson E. Using marketing principles for healthcare development. Quality in Health Care 1996;4:40-4.

10 Mittman BS, Tonesk X, Jacobson PD. Implementing clinical guidelines; social influence strategies and practitioner behaviour change. Quality Review Bulletin 1992;18:413-22.

11 Felton T, Lister G. Consider the evidence. Uxbridge: Coopers and Lybrand, 1996.

12 Prochaska JO, DiClemente CC, Norcross JC. In search of how people change: applications to addictive behaviors. $\mathrm{Am}$ Psychol 1992;47:1102-14.

13 National Health and Safety Executive, Northern and Yorkshire Region Research and Development Division directorate. Information need assessment report. Durham: NHS Executive, 1996

14 Davidson RA, Fletcher SW, Retchin S, et al. A nurse initiated reminder system for the periodic health Med 1984;144:2167-70.

15 Schwartz LM, Woloshin S, Welch HG. Trends in diagnostic testing following a national guideline for evaluation of dyspepsia. Arch Intern Med 1996;156:873-5.

16 Robertson N, Baker R, Hearnshaw H. Changing the clinical behaviour of doctors: a psychological framework. Quality in Health Care 1996;5:51-4.

17 Winett RA. A framework for health promotion and disease prevention programs. Am Psychol 1995;50:341-50.

18 Black ER, Anderson F, Enarson C, et al. Innovations in patient care: changing clinical practice and improving Meeting Abstract Book 1994;11:78-9.

19 North of England study of standards and performance in general practice. Overview of the study. Newcastle-upon Tyne: Cenpractice. Overview of the study. Newcast Health Services Research,1992.

20 Widger HN, Arai DA, Narasimhan K, et al. ACEP chest pain policy: emergency physician awareness. Ann Emerg

21 Stiell EG, McKnight RD, Greenberg GH, et al. Implementation of the Ottawa ankle rules. $₹ A M A$ 1994;271:827-32.

22 Donald A. Evidence-based medicine: a report from McMaster University Medical School and teaching hospitals. Oxford: NHS Executive, Anglia and Oxford, 1994.

23 Davis DA, Thomson MA, Oxman AD, et al. Evidence for the effectiveness of CME: a review of 50 randomised controlled trials. $¥ A M A$ 1992;268:1111-6.

24 Anderson FA, Wheeler HB, Goldberg RJ, et al. Changing clinical practice. Prospective study of the impact of continuing medical education and quality assurance continuing medical education and quality assurance
programs on use of prophylaxis for venous thromboemboprograms on use of prophylaxis for venous
lism. Arch Intern Med 1994;154:669-77.

25 Davis DA, Thomson MA, Oxman AD, et al. Changing physician performance: a systematic review of the effect of continuing medical education strategies. $\mathcal{F} A M A$ 1995;274 700-5

26 Walshe K, Ham C. Acting on the evidence: progress in the NHS; HSMC: University of Birmingham Report. Birmingham: University of Birmingham, 1997.

27 Winett RA, Altman DG, King A. Effective media campaigns to prevent the spread of HIV infection. Evaluation End Program Planning 1990;13:91-104.

28 Sibbald B, Roland M. Getting research into practice. fournal of Evaluation in Clinical Practice 1997;2:15-21.

29 Carney AJ, Carvey TA. Use of aspirin in secondary prevention of coronary heart disease is rising. BMF 1996;312:846.

30 Blake RR, Mouton JS. Making experience work. New York: McGraw-Hill, 1978 .

31 Firth-Cozens J, Handley S. Clinical audit: winning hearts and minds. Report to the National Health and Safety Executive Northern and Yorkshire Region. Leeds: University of Leeds, 1995.

32 Firth-Cozens J. Clinical effectiveness in Trusts. Report to the National Health and Safety Executive Northern and Yorkshir National Health and Safety Executive North
Region. Leeds: University of Leeds, 1997.

33 Firth-Cozens J. Stress, psychological problems, and clinical performance. In: Vincent C, Ennis M, Audley RJ, eds. Medical accidents. Oxford: Oxford University Press, 1993.

34 Warr P. Work, unemployment, and mental health. Oxford Oxford University Press, 1987.

35 Tunis SR, Hayward RSA, Wilson MC, et al. Internists' attitudes about clinical practice guidelines. Ann Intern Med 1994;120:956-63.

36 Chambless DL. Identification of empirically supported psychological interventions. Clinical Research Digest Supplemental Bulletin 1996;14.

37 Fisher JD, Misovich SJ. Changing AIDS risk behaviour. Psychol Bull 1992;111:455-74.

38 Firth-Cozens J. Depression in doctors. In: C Katona, MM Robertson, eds. Depression and physical illness. Chichester Wiley, 1997:95-111.

39 Karca A, Holbrook J. The Massachusetts emergency medicine risk management program. $Q R B$ 1991;17:287-92.

40 Wennberg JE, Barnes BA, Zubkoff $M$. Professional uncertainty and the problem of supplier-induced demand. $S o c$ Sci Med 1982;16:811-24.

41 Schwartz B. The creation and destruction of value. Am Psychol 1990;45:7-15.

42 Eisenberger R, Cameron J. Detrimental effects of reward. Am Psychol 1996;51:1153-66.

43 Firth-Cozens J. Tackling risk by changing behaviour. In: C Vincent, ed. Clinical risk management. London: BMJ, (1996). 
44 Ennis $M$, Clark A, Grudzinskas JG. Change in obstetric practice in response to fear of litigation in the British Isles. Lancet 1991;338:616.

45 Puri BK, Obaydi H, Singh I. Changes in the use of EEG following clinical audit. Br 7 Clin Pract 1996;50:52-3.

46 King AC, Blair SN, Bild DE, et al. Determinants of physical activity interventions in adults. Med Sci Sport Exerc activity interventions

47 Conroy M, Shannon W. Clinical guidelines: their implementation in general practice. $B r \mathcal{F}$ Gen Pract 1995;45:371-5.
48 Gray JD, Donaldson LJ. Improving the quality of health care through contracting: a study of health authority practice. Quality in Health Care 1996;5:201-5.

49 Murphy M, Dunning M. Implementing clinical effectiveness-is it time for a change of gear? British foumal of Health Care Management 1997;3:23-6.

50 Haynes RB, Sackett DL, Gray JMA, et al. Transferring evidence from research into practice: 1 . The role of clinical care research evidence in clinical decisions. Evidence-Based care research evidence
Medicine 1996;1:196-8. 